\title{
Review: antileukotrienes are less effective than inhaled corticosteroids in chronic asthma
}

\author{
Ducharme FM, Hicks GC.Anti-leukotriene agents compared to inhaled corticosteroids in the management of recurrent \\ and/or chronic asthma in adults and children. Cochrane Database Syst Rev 2002;(3):CD002314 (latest version 15 Jan 2002).
}

\author{
QUESTION: In patients with chronic asthma, are daily antileukotrienes (ALs) as safe \\ and effective as inhaled corticosteroids (ICSs)?
}

\section{Data sources}

Studies were identified by searching Medline (19662002), EMBASE/Excerpta Medica (1980-2002), CINAHL (1982-2002), the Cochrane Airways Group register of randomised controlled trials (RCTs), the Cochrane Controlled Trials Register (to January 2002); checking the reference lists of all relevant trials; contacting the pharmaceutical companies that manufacture ALs; and contacting colleagues and trialists in the field of paediatric asthma.

\section{Study selection}

Studies were selected if they were RCTs comparing daily oral ALs at usual licensed doses with any type of ICS in children $\geq 2$ years of age and adults with chronic asthma. Interventions had to be administered for $\geq 30$ days.

\section{Data extraction}

Data were extracted independently by 2 reviewers blinded to identifying information about the study on quality (randomisation, blinding, and description of withdrawals and dropouts), patient characteristics, drug type and dosage, and outcomes. The primary outcome was the rate of asthma exacerbations requiring systemic corticosteroids. Secondary outcomes were other indicators of exacerbation severity (hospital admissions), asthma control measures (change from baseline $\mathrm{FEV}_{1}$ and morning peak expiratory flow, symptom score, nocturnal awakenings, quality of life, and $\beta_{2}$ agonist use), adverse effects, and withdrawal rates.

\section{Main results}

14 trials met the inclusion criteria ( 8 full text, 1 in press, and 5 abstracts). 12 trials were done in adults, 1 was in adults and adolescents $(\geq 12 y)$, and 1 was in children (mean age $10 \mathrm{y}$ ). The duration of the intervention ranged from 4-37 weeks. The ALs tested were montelukast, pranlukast, and zafirlukast. 11 trials reported the primary outcome: more patients who received ALs than ICSs had an increased rate of asthma exacerbations (table). In 9 trials that reported the rate of exacerbation requiring hospital admission, the groups did not differ (relative risk [RR] 1.73, 95\% CI 0.64 to 4.73). All measures of asthma control at all follow up time points were worse in the AL group than the ICS group. Groups did not differ for adverse effects (11 trials, RR 1.0, CI 0.9 to 1.1). More withdrawals occurred in the AL group than the ICS group (10 trials, RR 1.3, CI 1.1 to 1.6).

\section{Conclusion}

In patients with chronic asthma, daily antileukotrienes are not as effective as inhaled corticosteroids and increase asthma exacerbations requiring systemic corticosteroids.

\section{COMMENTARY}

The meta-analysis by Ducharme and Hicks further strengthens the argument that ICSs should be used as first line treatment in mild persistent asthma. From pooled data of the 14 RCTs comparing ALs with ICSs as monotherapy in mild persistent asthma, ALs were less effective than ICSs in preventing asthma exacerbations requiring rescue oral steroids. However, no differences existed for more severe exacerbations requiring hospitalisation. This complements other data that suggest minimal further benefit in adding ALs to ICSs and that question allowing tapering of ICSs. ${ }^{1}$

The main issue to consider when interpreting data from clinical trials directly comparing ICSs and ALs is the large interpatient variability in response to all asthma medications, including ICSs. ${ }^{2}$ Group mean data favour ICSs over ALs, but the individual response to ALs varies. ${ }^{3}$ Such heterogeneity in response cannot currently be predicted from clinical parameters. As our understanding of pharmacogenetics improves, it may allow tailoring of medication regimens to suit individual patients according to probable response. Until then, ICSs at the minimum effective dose should be recommended as first line monotherapy of mild persistent asthma. ALs may be considered as an alternative to ICSs in patients with mild persistent asthma if compliance with inhaled medication is in question or if ICSs are associated with unacceptable oropharyngeal side effects.

Frank Thien, MD FRACP

Alfred Hospital and Monash University Melbourne, Victoria, Australia

1 Ducharme FM. Anti-leukotrienes as add-on therapy to inhaled glucocorticoids in patients with asthma: systematic review of current evidence. BMJ 2002;324:1545.

2 Szefler SJ, Martin RJ, King TS, et al. Significant variability in response to inhaled corticosteroids for persistent asthma. $J$

3 Malmstrom K, Rodriguez-Gomez G, Guerra J, et al. Oral montelukast, inhaled beclomethasone, and placebo for chronic asthma. A randomized, controlled trial. Montelukast/Beclomethasone Study Group. Ann Intern Med 1999;130:487-95.

Antileukotrienes (ALs) v inhaled corticosteroids (ICSs) for chronic asthma at 4-37 weeks*

\begin{tabular}{llllll} 
& \multicolumn{3}{l}{ Weighted event rates } & & \\
\cline { 2 - 3 } Outcome & ALs & ICSs & RRI (95\% CI) & NNH (CI) \\
\hline 1 asthma exacerbationrequiring systemic corticosteroids & $8.9 \%$ & $5.9 \%$ & $61 \%(15$ to 125$)$ & 34 (17 to 334$)$ \\
\hline
\end{tabular}

Source of funding: Fonds de la Santé du Québec Canada.

For correspondence: Dr F Ducharme, Montreal Children's Hospital, Montreal, Quebec, Canada. francine.ducharme@ muhc.mcgill.ca

Abstract and commentary also appear in ACP Journal Club.

*Abbreviations defined in glossary; RRI, NNH, and $\mathrm{Cl}$ calculated from data in article using a random effects model. 